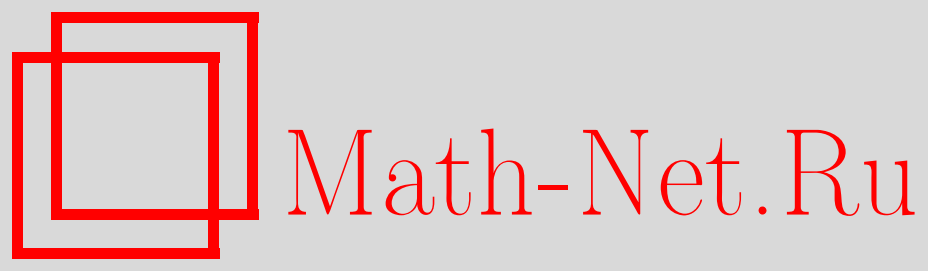

Л. А. Петров, Двухпараметрическое семейство бесконечномерных диффузий на симплексе Кингмана, Функи. анализ и его прил., 2009, том 43, выпуск 4, 45-66

DOI: https://doi.org/10.4213/faa2970

Использование Общероссийского математического портала MathNet.Ru подразумевает, что вы прочитали и согласны с пользовательским соглашением

http://www.mathnet.ru/rus/agreement

Параметры загрузки:

IP : 52.23 .180 .231

26 апреля 2023 г., 05:02:51

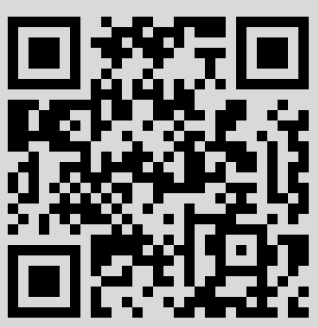




\title{
Двухпараметрическое семейство бесконечномерных диффузий на симплексе Кингмана
}

\author{
(C) 2009. Л. А. Петров
}

В данной работе построено двухпараметрическое семейство диффузионных процессов $\mathbf{X}_{\alpha, \theta}$ на симплексе Кингмана, состоящем из невозрастающих последовательностей неотрицательных чисел с суммой не больше единицы. Процессы на этом симплексе возникают как пределы марковских цепей на разбиениях натуральных чисел.

В случае $\alpha=0$ наш процесс совпадает с популяционно-генетической динамической моделью бесконечного количества нейтральных аллелей, построенной Этье и Куртцом (1981). Двухпараметрический случай, по-видимому, не имеет популяционногенетической интерпретации. В настоящей работе обобщаются основные результаты Этье и Куртца на случай двух параметров. А именно, мы показываем, что единственной инвариантной мерой процесса $\mathbf{X}_{\alpha, \theta}$ является (двухпараметрическая) мера Пуассона-Дирихле $\mathrm{PD}(\alpha, \theta)$, причем процесс обратим и эргодичен относительно нее. Мы вычисляем спектр его генератора. Также оказывается, что диффузии РайтаФишера на конечномерных симплексах возникают как частный случай процесса $\mathbf{X}_{\alpha, \theta}$ при определенных вырожденных значениях параметров.

\section{Введение}

Основной результат данной работы состоит в построении двухпараметрического семейства марковских процессов $\mathbf{X}_{\alpha, \theta}(t)$ на бесконечномерном симплексе Кингмана

$$
\bar{\nabla}_{\infty}=\left\{x=\left(x_{1}, x_{2}, \ldots\right) \in[0,1]^{\infty}: x_{1} \geqslant x_{2} \geqslant \ldots \geqslant 0, \sum_{i=1}^{\infty} x_{i} \leqslant 1\right\} .
$$

В топологии покоординатной сходимости $\bar{\nabla}_{\infty}$ является метризуемым сепарабельным компактом.

Пусть $C\left(\bar{\nabla}_{\infty}\right)$ - алгебра непрерывных функций на $\bar{\nabla}_{\infty}$ с поточечными операциями и супремум-нормой. Определим в ней плотное подпространство $\mathscr{F}:=$ $\mathbb{R}\left[q_{1}, q_{2}, \ldots\right]$, порожденное (как коммутативная алгебра с единицей) непрерывными алгебраически независимыми функциями $q_{k}(x):=\sum_{i=1}^{\infty} x_{i}^{k+1}, k=1,2, \ldots$, $x \in \bar{\nabla}_{\infty}$.

Для любых $0 \leqslant \alpha<1$ и $\theta>-\alpha$ определим оператор $A: \mathscr{F} \rightarrow \mathscr{F}$, который может быть записан как формальный дифференциальный оператор второго порядка по образующим алгебры $\mathscr{F}$ :

$$
\begin{aligned}
A=\sum_{i, j=1}^{\infty}(i+1)(j+1)\left(q_{i+j}\right. & \left.-q_{i} q_{j}\right) \frac{\partial^{2}}{\partial q_{i} \partial q_{j}} \\
& +\sum_{i=1}^{\infty}\left[-(i+1)(i+\theta) q_{i}+(i+1)(i-\alpha) q_{i-1}\right] \frac{\partial}{\partial q_{i}},
\end{aligned}
$$


а также (с некоторыми оговорками, см. замечания 5.3 и 5.4 ниже) как дифференциальный оператор в естественных координатах:

$$
A=\sum_{i=1}^{\infty} x_{i} \frac{\partial^{2}}{\partial x_{i}^{2}}-\sum_{i, j=1}^{\infty} x_{i} x_{j} \frac{\partial^{2}}{\partial x_{i} \partial x_{j}}-\sum_{i=1}^{\infty}\left(\theta x_{i}+\alpha\right) \frac{\partial}{\partial x_{i}}
$$

Следует особо отметить, что оператор $A$ определен только на $\mathscr{F}$, и применение правых частей формул (2) или (3) к другим функциям напрямую требует осторожности.

\section{Основная теорема. (1) Oператор $A$ замъкаем в $C\left(\bar{\nabla}_{\infty}\right)$.}

(2) Его замыкание $\bar{A}$ порождает дифбузионный процесс $\left\{\mathbf{X}_{\alpha, \theta}(t)\right\}_{t \geqslant 0}$ на $\bar{\nabla}_{\infty}$ (под дифбузионным процессом понимается строго марковский процесс с непрерывными траекториями).

(3) Двухпараметрическая мера Пуассона-Дирихле $\mathrm{PD}(\alpha, \theta)$ на $\bar{\nabla}_{\infty}$ является единственной инвариантной вероятностной мерой процесса $\mathbf{X}_{\alpha, \theta}(t)$. Проиесс обратим относительно этой меры.

(4) Спектр генератора $\bar{A}$ проиесса $\mathbf{X}_{\alpha, \theta}(t)$ вычислен в явном виде в разд. 4.2 ниже. Благодаря наличию спектральной щели $\mathbf{X}_{\alpha, \theta}(t)$ эргодичен по отношению к мере $\operatorname{PD}(\alpha, \theta)$.

Пусть $0 \leqslant \alpha<1$ и $\theta>-\alpha$ фиксированы. Процесс $\mathbf{X}_{\alpha, \theta}(t)$ на симплексе $\bar{\nabla}_{\infty}$ строится как предел конечных марковских цепей с растущим числом состояний. Пространство состояний $n$-й цепи представляет собой множество всех разбиений числа $n$ на натуральные слагаемые и обозначается через $\mathbb{K}_{n}$. Каждое $\mathbb{K}_{n}$ снабжено вероятностной мерой $M_{n}$ (также зависящей от $\alpha$ и $\theta$ ), которая выписывается в явном виде. Совокупность $\left\{M_{n}\right\}$ является структурой разбиений ${ }^{1)}$. В [19] установлено взаимно однозначное соответствие между структурами разбиений и вероятностными мерами на $\bar{\nabla}_{\infty}$. Мера Пуассона-Дирихле $\mathrm{PD}(\alpha, \theta)$ соответствует двухпараметрической структуре разбиений Ювенса-Питмана $\left\{M_{n}\right\}$. При $\alpha=0$ эта структура разбиений была введена в работе [7], двухпараметрическое обобщение принадлежит Питману [22]. Частный случай системы $\left\{M_{n}\right\}$ при $\alpha=0, \theta=1$ рассматривался в работах [28], [13] в связи с предельным поведением некоторых функционалов на симметрической группе $\mathfrak{S}_{n}$ при $n \rightarrow \infty$, там же изучалась мера $\mathrm{PD}(0,1)$. Двухпараметрические структуры разбиений Ювенса-Питмана изучались в работах [23], [25], [11], [26], [3] и многих других. См. также библиографию в [25].

Стоит отметить, что первый пример марковского процесса на $\bar{\nabla}_{\infty}$, для которого двухпараметрическая мера Пуассона-Дирихле является единственной инвариантной симметризующей вероятностной мерой, построен в работе [1]. Также в недавней работе [10] рассматриваются диффузии на неупорядоченном бесконечномерном симплексе, сохраняющие GEM-распределение (Griffiths-EngenMcCloskey, см. [25]) на нем. При упорядочении координат GEM-распределение становится мерой Пуассона-Дирихле.

Случай $\alpha=0$ относительно хорошо изучен и имеет приложения в популяционной генетике. Мера $\mathrm{PD}(0, \theta)$ появилась в работе [17]. Процесс $\mathbf{X}_{0, \theta}(t)$ был

1) Термин «partition structure» был введен в [19]. Общепринятого перевода на русский язык, по-видимому, не существует. В более общей ситуации используется также термин «coherent system» (см., например, [16], [2]). 
построен в [4] как предел диффузионных процессов на конечномерных симплексах растущей размерности. В разд. 5.3 будет показано, что эти конечномерные диффузии являются частным случаем процесса $\mathbf{X}_{\alpha, \theta}(t)$ при значениях параметров $\alpha=-\beta<0, \theta=N \beta, N=2,3, \ldots$ Также легко видеть, что при $\alpha=0$ оператор $A$, записанный в форме $(3)$, совпадает с удвоенным оператором из формулы (2.10) работы [4].

Кроме того, в [4] доказано, что процесс $\mathbf{X}_{0, \theta}(t)$ возникает как предельный в популяционной модели типа Морана (об этой модели см. также, например, [29] и [14, модель II]). Модель типа Морана представляет собой последовательность марковских цепей на разбиениях, которые отличаются от рассматриваемых в данной работе, однако имеют тот же предел.

Процесс $\mathbf{X}_{0, \theta}(t)$ (при $\alpha=0$ ) называется моделью бесконечного количества нейтральных аллелей ${ }^{1)}$, он изучался также в [6], [27] и других работах. Результаты данной работы обобщают некоторые результаты из [4] и [6] на случай $\alpha \neq 0$. В этом случае, по-видимому, теряется связь модели $\mathbf{X}_{\alpha, \theta}(t)$ с популяционной генетикой - ни для конечных марковских цепей, ни для нашего процесса на $\bar{\nabla}_{\infty}$ такой интерпретации не известно.

В настоящей работе построение процесса $\mathbf{X}_{\alpha, \theta}(t)$ на $\bar{\nabla}_{\infty}$ использует только понятия структуры разбиений и марковских цепей вверх/вниз. Впервые в контексте, рассматриваемом в данной работе, цепи вверх/вниз появились в [8]. Они также изучались в работах [2], [9]. Постановка задачи и общий подход к ее решению инспирированы работой [2]. Однако конкретные вычисления здесь осуществлены другим способом. Роль, которую в [2] играют функции Шура, в нашей ситуации выполняют мономиальные симметрические функции. Они проще функций Шура, и результат достигается более простыми средствами. Дополнительно к результатам, аналогичным результатам из [2], удается вычислить оператор $A$ в естественных координатах (3) и явно описать процесс $\mathbf{X}_{\alpha, \theta}(t)$ при $\alpha=-\beta<0$ и $\theta=N \beta$. Приведем краткий план работы.

В разд. 1.1 введены основные обозначения, связанные с разбиениями. В разд. 1.2 приводятся определения структур разбиений и связанных с ними марковских цепей вверх/вниз. В разд. 1.3 приводятся определение и некоторые свойства структур разбиений Ювенса-Питмана.

В $\S 2$ рассматриваются некоторые свойства симметрических функций от координат $\left(x_{1}, x_{2}, \ldots\right)$ точки $x \in \bar{\nabla}_{\infty}$. Симметрические функции образуют алгебру $\mathscr{F}$, определенную выше. В этих терминах формулируется теорема Кингмана о взаимно однозначном соответствии между структурами разбиений и вероятностными мерами на $\bar{\nabla}_{\infty}$.

В $\S 3$ получено явное выражение для действия переходных операторов $T_{n}$ марковских цепей вверх/вниз на симметрические функции от компонент $\lambda_{1}, \lambda_{2}, \ldots$ разбиений $\lambda \in \mathbb{K}_{n}$.

В разд. 4.1 совершается предельный переход от цепей вверх/вниз к процессу $\mathbf{X}_{\alpha, \theta}(t)$ на $\bar{\nabla}_{\infty}$. Сначала, используя связь симметрических функций от компонент разбиения с симметрическими функциями на $\bar{\nabla}_{\infty}$, мы явно находим предел операторов $n^{2}\left(T_{n}-\mathbf{1}\right)$, который равен оператору $A: \mathscr{F} \rightarrow \mathscr{F}$, выписанному выше. Затем, используя абстрактные результаты книги [5], мы устанавливаем сходимость дискретных полугрупп $\left\{\mathbf{1}, T_{n}, T_{n}^{2}, \ldots\right\}$ к непрерывной полугруппе

1) The infinitely many neutral alleles diffusion model (англ.) 
$\{T(t)\}_{t \geqslant 0}$ процесса $\mathbf{X}_{\alpha, \theta}(t)$, порожденной замыканием $\bar{A}$ этого оператора $A$. В разд. 4.2 приведены остальные результаты основной теоремы, сформулированной выше, кроме непрерывности траекторий процесса $\mathbf{X}_{\alpha, \theta}(t)$.

В разд. 5.1 получена формула (3). Там также объясняется, почему правую часть этой формулы нельзя понимать буквально. В разд. 5.2 получена формула (2) для оператора $A$. Используя это выражение, удается доказать непрерывность траекторий рассматриваемого процесса на $\bar{\nabla}_{\infty}$. В разд. 5.3 сделано замечание о вырожденных значениях параметров $\alpha=-\beta, \theta=N \beta$, при которых вместо диффузии на $\bar{\nabla}_{\infty}$ в пределе марковских цепей вверх/вниз получается диффузия на $(N-1)$-мерном симплексе, совпадающая с рассмотренной в [4].

Автор глубоко признателен Г. И. Ольшанскому за постановку задачи, внимание к работе и ценные замечания.

\section{§1. Структуры разбиений Ювенса-Питмана}

1.1. Обозначения. Приведем основные комбинаторные обозначения, которые используются на протяжении всей работы.

Под разбиением будем понимать невозрастающую последовательность неотрицательных целых чисел

$$
\lambda=\left(\lambda_{1}, \lambda_{2}, \ldots, \lambda_{\ell}, 0,0, \ldots\right), \quad \lambda_{1} \geqslant \lambda_{2} \geqslant \ldots \geqslant \lambda_{\ell}>0, \lambda_{i} \in \mathbb{Z}_{>0},
$$

в которой содержится лишь конечное число ненулевых элементов.

Разбиения отождествляются с диаграммами Юнга, см. [20], и обозначаются теми же буквами. Число клеток в $\lambda$ обозначается через $|\lambda|=\lambda_{1}+\cdots+\lambda_{\ell}$. Число строк в $\lambda$ называется длиной диаграммы и обозначается через $\ell(\lambda)$. Пусть также $\varnothing-$ пустая диаграмма Юнга.

Если диаграмма $\lambda$ получена из диаграммы $\mu$ путем добавления одной клетки, то будем писать $\mu \nearrow \lambda$ или, что эквивалентно, $\lambda \searrow \mu$. Клетку, которой отличаются $\mu$ и $\lambda$, обозначим через $\lambda / \mu$.

Для любой клетки $\square$ на плоскости обозначим через $\mathrm{r}(\square)$ номер столбца, в котором она лежит, считая слева направо.

Фиксируем произвольную непустую диаграмму $\lambda$. Отметим следующие важные свойства. Во-первых, для любого $i=1, \ldots, \ell(\lambda)$ существует единственная клетка с $\mathrm{r}(\square)=\lambda_{i}$, обозначаемая через $\square\left(\lambda_{i}\right)$, которую можно убрать из $\lambda$, так что снова получится диаграмма Юнга. Полученную диаграмму обозначим через $\lambda-\square\left(\lambda_{i}\right)$. Так описываются все диаграммы $\mu$, такие, что $\mu \nearrow \lambda$. Во-вторых, для любого $i=1, \ldots, \ell(\lambda)$ существует единственная клетка с $\mathrm{r}(\square)=\lambda_{i}+1$, обозначаемая через $\widehat{\square}\left(\lambda_{i}+1\right)$, которую можно прибавить к $\lambda$, так что снова получится диаграмма Юнга. Полученную диаграмму обозначим через $\lambda+\widehat{\square}\left(\lambda_{i}+1\right)$. Все диаграммы $\nu, \nu \searrow \lambda$, имеют либо вид $\lambda+\widehat{\square}\left(\lambda_{i}+1\right)$, либо вид $\lambda+\widehat{\square}(1)$, где $\lambda+\widehat{\square}(1)-$ диаграмма, полученная из $\lambda$ добавлением строки, состоящей из одной клетки.

Для натурального $k$ через $[\lambda: k]$ обозначим число строк в $\lambda$ длины $k$. Это неотрицательное целое число. Для диаграмм Юнга $\mu, \lambda$, таких, что $|\lambda|=|\mu|+1$, положим

$$
\kappa(\mu, \lambda):= \begin{cases}{[\lambda: \mathrm{r}(\lambda / \mu)],} & \text { если } \mu \nearrow \lambda, \\ 0 & \text { в противном случае. }\end{cases}
$$


Все диаграммы Юнга организованы в градуированное множество

$$
\mathbb{K}:=\bigsqcup_{n=0}^{\infty} \mathbb{K}_{n},
$$

где $\mathbb{K}_{n}=\{\lambda:|\lambda|=n\}, n \in \mathbb{Z}_{>0}, \mathbb{K}_{0}=\{\varnothing\}$. На этом множестве вводится структура градуированного графа с ребрами только между соседними «этажами» $\mathbb{K}_{n}$ и $\mathbb{K}_{n+1}$. Между $\mu \in \mathbb{K}_{n}$ и $\lambda \in \mathbb{K}_{n+1}$ проводится $\kappa(\mu, \lambda)$ ребер. Считаем, что они ориентированы в направлении от $\mathbb{K}_{n} \mathrm{~K} \mathbb{K}_{n+1}$. Этот граф (называемый в литературе графом Кингмана) отличен от графа Юнга, в котором проведены те же ребра, только однократные.

Обозначим через $g(\mu, \lambda)$ количество ориентированных путей от $\mu$ до $\lambda$ в графе $\mathbb{K}$. Ясно, что это число отлично от нуля, только если $\mu \subset \lambda$ как диаграммы (множества клеток на плоскости). Положим $g(\lambda):=g(\varnothing, \lambda)$. Оказывается, что $g(\lambda)=|\lambda| ! /\left(\lambda_{1} ! \cdots \lambda_{\ell(\lambda)} !\right)$.

Также нам понадобятся символы Похгаммера

$$
(a)_{k}:=a(a+1) \cdots(a+k-1), \quad k=1,2, \ldots, \quad(a)_{0}:=1
$$

и факториальные степени

$$
a^{\downarrow k}:=a(a-1) \cdots(a-k+1), \quad k=1,2, \ldots, \quad a^{\downarrow 0}:=1 .
$$

1.2. Марковские цепи вверх/вниз. Структура графа на $\mathbb{K}$ важна, однако в данной работе она используется только для того, чтобы ниже применить рекуррентные соотношения (7) для величин $g(\mu, \lambda)$.

Одним из важнейших рассматриваемых объектов являются марковские иепи вверx/вниз. Они изучались в работах [2], [8], [9] $]^{1)}$. Для их определения далее используется формализм из [2, §1].

Переходной функиией вниз для $\mu, \lambda \in \mathbb{K}$, таких, что $|\lambda|=|\mu|+1$, называется

$$
p^{\downarrow}(\lambda, \mu):=\frac{g(\mu)}{g(\lambda)} \kappa(\mu, \lambda) .
$$

Легко проверить следующие ее свойства:

- $p^{\downarrow}(\lambda, \mu) \geqslant 0$ для всех $\mu, \lambda \in \mathbb{K}$, таких, что $|\lambda|=|\mu|+1$;

- $p^{\downarrow}(\lambda, \mu)$ отлична от нуля только для $\mu \nearrow \lambda$;

- если $|\lambda|=n \geqslant 1$, то $\sum_{\mu:|\mu|=n-1} p^{\downarrow}(\lambda, \mu)=1$.

Замечание 1.1. Отметим, что одно и то же семейство переходных функций вниз может получиться при разных функциях кратности $\kappa(\cdot, \cdot)($ и соответственно разных величинах $g(\cdot, \cdot)$, которые определяются по кратностям). В работе [15], помимо кратностей (4), рассматриваются еще две целочисленные функции кратности, которые приводят к тем же переходным функциям вниз (такие функции кратности называются подобньми). Иногда в литературе под графом Кингмана понимают граф с теми же вершинами, но другой функцией кратности, которая подобна функции (4).

1) В [8], [9] были введены и изучались цепи вниз/вверх; их отличие от цепей вверх/вниз незначительно. 
Структурой разбиений на $\left(\mathbb{K}, p^{\downarrow}\right)$ называется семейство $\left\{M_{n}\right\}_{n \geqslant 0}$, где $M_{n}$ вероятностная мера на $\mathbb{K}_{n}, n \geqslant 0$, согласованное с переходной функцией вниз в том смысле, что

$$
M_{n}(\mu)=\sum_{\lambda: \lambda \backslash \mu} M_{n+1}(\lambda) p^{\downarrow}(\lambda, \mu) \quad \text { для всех } n \in \mathbb{Z}_{\geqslant 0} \text { и } \mu \in \mathbb{K}_{n} .
$$

Здесь $M_{n}(\mu)$ означает меру одноточечного множества $\{\mu\}$.

Фиксируем структуру разбиений $\left\{M_{n}\right\}$. Переходной функиией вверх для $\lambda, \nu \in \mathbb{K}$, таких, что $|\nu|=|\lambda|+1$ и $M_{n}(\lambda) \neq 0$, называется

$$
p^{\uparrow}(\lambda, \nu):=\frac{M_{n+1}(\nu)}{M_{n}(\lambda)} p^{\downarrow}(\nu, \lambda), \quad \lambda \nearrow \nu .
$$

Эта функция зависит от конкретной структуры разбиений. Кроме того, семейство $\left\{M_{n}\right\}$ согласовано с $p^{\uparrow}$ (в смысле, похожем на $\left.(5)\right)$ :

$$
M_{n+1}(\nu)=\sum_{\lambda: \lambda \nearrow \nu, \lambda \in \operatorname{supp}\left(M_{n}\right)} M_{n}(\lambda) p^{\uparrow}(\lambda, \nu) \quad \text { для всех } n \in \mathbb{Z}_{\geqslant 0} \text { и } \nu \in \mathbb{K}_{n+1} \text {. }
$$

Пусть $\left\{M_{n}\right\}$ - структура разбиений и $M_{n}(\lambda)>0$ для всех $n \geqslant 0$ и всех $\lambda \in \mathbb{K}_{n}$. Определим на каждом $\mathbb{K}_{n}, n \geqslant 1$, марковскую цепь $T_{n}$ с переходной матрицей

$$
T_{n}(\lambda, \tilde{\lambda}):=\sum_{\nu:|\nu|=n+1} p^{\uparrow}(\lambda, \nu) p^{\downarrow}(\nu, \tilde{\lambda}), \quad \lambda, \tilde{\lambda} \in \mathbb{K}_{n} .
$$

Это композиция переходных функций - сначала вверх из $\mathbb{K}_{n}$ в $\mathbb{K}_{n+1}$ и затем обратно в $\mathbb{K}_{n}$. Из определений, приведенных выше, следует, что $M_{n}$ является стационарным распределением для $T_{n}$. Можно легко показать, что матрица $M_{n}(\lambda) T_{n}(\lambda, \widetilde{\lambda})$ симметрична относительно замены $\lambda \leftrightarrow \widetilde{\lambda}$, а это означает, что цепь $T_{n}$ обратима относительно $M_{n}$.

1.3. Структуры разбиений Ювенса-Питмана. В данной работе рассматривается специальное двухпараметрическое семейство структур разбиений Ювенса-Питмана.

Пусть $\alpha$ и $\theta-$ некоторые параметры. Положим

$$
M_{n}(\lambda):=\frac{n !}{(\theta)_{n}} \cdot \frac{\theta(\theta+\alpha) \cdots(\theta+(\ell(\lambda)-1) \alpha)}{\prod_{k=1}^{\infty}[\lambda: k] ! \cdot \prod_{i=1}^{\ell(\lambda)} \lambda_{i} !} \prod_{\substack{\square \in \lambda \\ \mathrm{r}(\square) \geqslant 2}}(\mathrm{r}(\square)-1-\alpha)
$$

для всех $n \in \mathbb{Z}_{\geqslant 0}$ и $\lambda \in \mathbb{K}_{n}$.

Можно показать, что для $M_{n}$ выполнено условие (5) для всех значений параметров $\alpha$ и $\theta$, при которых $M_{n}$ определены, а также что при этом

$$
\sum_{\lambda:|\lambda|=n} M_{n}(\lambda)=1 \quad \text { для всех } n \in \mathbb{Z}_{\geqslant 0} .
$$

Кроме того, можно проверить, что выражения $M_{n}(\lambda)$ неотрицательны для всех $n \geqslant 0$ и $\lambda \in \mathbb{K}_{n}$, если и только если

- (основная серия параметров) $0 \leqslant \alpha<1$ и $\theta>-\alpha$;

- (вырожденная серия параметров) $\alpha=-\beta<0$ и $\theta=N \beta$ для некоторого $N=2,3, \ldots$.

В основной серии носитель каждой меры $M_{n}$ есть все множество $\mathbb{K}_{n}$, а в вырожденной серии $M_{n}(\lambda)>0$ только для $\lambda$ длины $\leqslant N$. Таким образом, когда 
параметры лежат в основной или вырожденной серии, $\left\{M_{n}\right\}_{n \geqslant 0}$ является структурой разбиений. Она называется структурой разбиений Ювенса-Питмана. Легко показать, что переходная функция вверх для этой структуры разбиений имеет вид

$$
p^{\uparrow}(\lambda, \nu)= \begin{cases}\frac{\lambda_{i}-\alpha}{n+\theta}\left[\lambda: \lambda_{i}\right], & \text { если } \nu=\lambda+\widehat{\square}\left(\lambda_{i}+1\right) \text { для } 1 \leqslant i \leqslant \ell(\lambda), \\ \frac{\theta+\ell(\lambda) \alpha}{n+\theta}, & \text { если } \nu=\lambda+\widehat{\square}(1), \\ 0 & \text { в противном случае, }\end{cases}
$$

где $|\lambda|=n \in \mathbb{Z}_{\geqslant 0}$.

Всюду далее, кроме разд. 5.3, предполагается, что параметры $\alpha$ и $\theta$ лежат в основной серии. Тогда, как объясняется в разд. 1.2, можно рассматривать цепи вверх/вниз на всем $\mathbb{K}_{n}$ (для $n \geqslant 1$ ), поскольку мера $M_{n}$ всюду положительна на $\mathbb{K}_{n}$.

\section{§2. Симметрические функции и симплекс Кингмана}

2.1. Симметрические функции. Пусть $\Lambda$ - (вещественная) алгебра симметрических функций от формальных переменных $y_{1}, y_{2}, \ldots$ [20, гл. I, §2]. В дальнейшем нам понадобятся следующие понятия:

Суммы Нъютона $p_{k}=\sum_{i=1}^{\infty} y_{i}^{k}, k=1,2, \ldots$ Эти элементы являются алгебраически независимыми образующими $\Lambda$ как коммутативной алгебры с единицей: $\Lambda=\mathbb{R}\left[p_{1}, p_{2}, p_{3}, \ldots\right]$.

Мономиальные функиии $m_{\lambda}, \lambda \in \mathbb{K}$, которые определяются как $\sum y_{i_{1}}^{\lambda_{1}} \cdots y_{i_{\ell}(\lambda)}^{\lambda_{\ell(\lambda)}}$, где суммирование ведется по всем различным мономам, а индексы $i_{1}, \ldots, i_{\ell}(\lambda)$ пробегают попарно различные значения от 1 до $\infty$, а также их кратные ${ }^{1)}$ вида $\mathbf{m}_{\lambda}:=\left(\prod_{k \geqslant 1}[\lambda: k] !\right) m_{\lambda}$, которые можно рассматривать как аналогичные суммы $\sum y_{i_{1}}^{\lambda_{1}} \ldots y_{i_{\ell}(\lambda)}^{\lambda_{\ell(\lambda)}}$ по всем наборам попарно различных индексов $i_{1}, \ldots, i_{\ell(\lambda)}$ от 1 до $\infty$. Рассмотрение функций $\mathbf{m}_{\lambda}$ вместо $m_{\lambda}$ в некоторых случаях удобней. Каждая из систем $\left\{m_{\lambda}\right\}_{\lambda \in \mathbb{K}}$ и $\left\{\mathbf{m}_{\lambda}\right\}_{\lambda \in \mathbb{K}}$ является базисом для $\Lambda$ как линейного пространства над $\mathbb{R}$.

Факториальные функиии $m_{\lambda}^{*}$ и $\mathbf{m}_{\lambda}^{*}, \lambda \in \mathbb{K}$, которые получаются из $m_{\lambda}$ и $\mathbf{m}_{\lambda}$ соответственно заменой каждой степени переменного $y_{i}^{k}$ на факториальную степень $y_{i}^{\downarrow k}$. Однородные части функций $m_{\lambda}^{*}$ и $\mathbf{m}_{\lambda}^{*}$ максимальной суммарной степени $|\lambda|$ равны соответственно $m_{\lambda}$ и $\mathbf{m}_{\lambda}$. Каждая из систем $\left\{m_{\lambda}^{*}\right\}_{\lambda \in \mathbb{K}}$ и $\left\{\mathbf{m}_{\lambda}^{*}\right\}_{\lambda \in \mathbb{K}}$ также является базисом $\Lambda$ как линейного пространства над $\mathbb{R}$.

Пусть $I:=\left(p_{1}-1\right) \Lambda$ есть главный идеал в $\Lambda$, порожденный элементом $p_{1}-1$. Положим $\Lambda^{\circ}:=\Lambda / I$. Каждому элементу $f \in \Lambda$ соответствует образ в $\Lambda^{\circ}$, обозначим его через $f^{\circ}$. В частности, $p_{1}^{\circ}=1$ и $\Lambda^{\circ}$ является коммутативной алгеброй с единицей, свободно порожденной элементами $p_{k}^{\circ}, k=2,3, \ldots$ Кроме того,

$$
\Lambda=\mathbb{R}\left[p_{1}, p_{2}, p_{3} \ldots\right]=I \oplus \mathbb{R}\left[p_{2}, p_{3}, \ldots\right],
$$

1) Называемые также аугментированными мономиальными функциями. 
а значит, $\Lambda^{\circ} \cong \mathbb{R}\left[p_{2}, p_{3}, \ldots\right]$. Легко проверить, что базис последней алгебры над $\mathbb{R}$-это $\left\{m_{\lambda}\right\}_{\lambda \in \mathbb{K},[\lambda: 1]=0}$. Поэтому $\mathbb{R}$-базисом в $\Lambda^{\circ}$ является система $\left\{m_{\lambda}^{\circ}\right\}_{\lambda \in \mathbb{K},[\lambda: 1]=0} \cdot$

2.2. Симплекс Кингмана и моментные координаты. Во введении был определен симплекс Кингмана (1). Это компактное метризуемое и сепарабельное пространство в топологии покоординатной сходимости, которая и будет использоваться. В нем выделим плотное подмножество $\nabla_{\infty}:=\left\{x=\left(x_{1}, x_{2}, \ldots\right) \in\right.$ $\left.\bar{\nabla}_{\infty}: \sum_{i=1}^{\infty} x_{i}=1\right\}$. Эти обозначения следуют работе [4]. Через $C\left(\bar{\nabla}_{\infty}\right)$ обозначим алгебру вещественных непрерывных на $\bar{\nabla}_{\infty}$ функций (относительно поточечных операций) с супремум-нормой.

Каждой точке $x \in \bar{\nabla}_{\infty}$ соответствует вероятностная мера

$$
\nu_{x}:=\sum_{i=1}^{\infty} x_{i} \delta_{x_{i}}+\gamma(x) \delta_{0}
$$

на отрезке $[0,1]$, где $\delta_{s}$ означает меру Дирака в точке $s$ и $\gamma(x):=1-\sum_{i=1}^{\infty} x_{i}$. Через $q_{k}(x)$ обозначим $k$-й момент меры $\nu_{x}$ :

$$
q_{k}(x):=\int_{0}^{1} u^{k} \nu_{x}(d u)=\sum_{i=1}^{\infty} x_{i}^{k+1}, \quad k=1,2, \ldots .
$$

Это непрерывные функции на $\bar{\nabla}_{\infty}$, поскольку для каждого $i=1,2, \ldots$ выполнено $x_{i} \leqslant i^{-1}$. Стоит отметить, что функция $\gamma(x)$ не является непрерывной на $\bar{\nabla}_{\infty}$. Кроме того, $q_{k}(x), k \geqslant 1$, разделяют точки симплекса $\bar{\nabla}_{\infty}$ (поскольку мера на $[0,1]$ однозначно определяется своими моментами) и являются алгебраически независимыми. По аналогии с работой [2] $q_{1}(x), q_{2}(x), \ldots$ назовем моментнъми координатами точки $x \in \bar{\nabla}_{\infty}$.

Пусть $\mathscr{F}=\mathbb{R}\left[q_{1}, q_{2}, \ldots\right]$ - коммутативная алгебра с единицей, порожденная моментными координатами. По теореме Стоуна-Вейерштрасса она является плотной подалгеброй в $C\left(\bar{\nabla}_{\infty}\right)$.

2.3. Симметрические функции на симплексе. Теорема Кингмана. Соответствие $p_{2}^{\circ} \rightarrow q_{1}(x), p_{3}^{\circ} \rightarrow q_{2}(x), \ldots$ устанавливает изоморфизм алгебры $\Lambda^{\circ}$, введенной в разд. 2.1, на алгебру $\mathscr{F}$ из разд. 2.2. Таким образом, каждому элементу $f^{\circ} \in \Lambda^{\circ}$ соответствует непрерывная функция на $\bar{\nabla}_{\infty}$, которую мы обозначим через $f^{\circ}(x)$. В частности, $p_{k}^{\circ}(x)=\sum_{i=1}^{\infty} x_{i}^{k}, k=2,3, \ldots$, и $p_{1}^{\circ}(x) \equiv 1$.

Приведем формулировку предельной теоремы Кингмана для структур разбиений. Рассмотрим для $n=1,2, \ldots$ вложения

$$
\iota_{n}: \mathbb{K}_{n} \hookrightarrow \bar{\nabla}_{\infty}, \quad \iota_{n}:\left(\lambda_{1}, \ldots, \lambda_{\ell}\right) \mapsto\left(\frac{\lambda_{1}}{n}, \ldots, \frac{\lambda_{\ell}}{n}, 0,0, \ldots\right) \in \bar{\nabla}_{\infty},
$$

где $\lambda=\left(\lambda_{1}, \ldots, \lambda_{\ell}\right) \in \mathbb{K}_{n}$. Следующее замечание будет полезно в $\S 4$.

Замечание 2.1. Образы $\iota_{n}\left(\mathbb{K}_{n}\right)$ аппроксимируют пространство $\bar{\nabla}_{\infty}$ в том смысле, что каждое открытое множество в $\bar{\nabla}_{\infty}$ имеет непустое пересечение с $\iota_{n}\left(\mathbb{K}_{n}\right)$ для всех достаточно больших $n$.

Теорема 2.2 ([19], [16]). Для любой структурь разбиений $\left\{M_{n}\right\}$ на $\mathbb{K}$ существует борелевская вероятностная мера $P$ на $\bar{\nabla}_{\infty}$ (называемая граничной 
мерой этой структуры разбиений), такая, что

$$
P=\lim _{n \rightarrow \infty} \iota_{n}\left(M_{n}\right) .
$$

И обратно, любая структура разбиений может быть восстановлена по своей граничной мере следующим образом:

$$
M_{n}(\lambda)=g(\lambda) \int_{\bar{\nabla}_{\infty}} m_{\lambda}^{\circ}(x) P(d x) \quad \text { для всех } \lambda \in \mathbb{K}_{n} .
$$

Таким образом, структурь разбиенийна $\mathbb{K}$ и борелевские вероятностнъе меръ на $\bar{\nabla}_{\infty}$ находятся во взаимно однозначном соответствии.

Структуре разбиений Ювенса-Питмана (разд. 1.3) с параметрами $\alpha$ и $\theta$, лежащими в основной или вырожденной серии, соответствует хорошо известная мера Пуассона-Дирихле $\mathrm{PD}(\alpha, \theta)$. Мера $\mathrm{PD}(\alpha, \theta)$ сосредоточена на плотном подмножестве $\nabla_{\infty} \subset \bar{\nabla}_{\infty}$ (определение $\nabla_{\infty}$ см. в разд. 2.2), см., например, [21], [24].

\section{§3. Действие переходных операторов цепей вверх/вниз на симметрические функции}

В этом параграфе предполагается, что параметры $\alpha$ и $\theta$ лежат в основной серии. Сначала введем некоторые дополнительные обозначения.

Для любого множества $\mathscr{X}$ обозначим через $\operatorname{Fun}(\mathscr{X})$ алгебру вещественных функций на $\mathscr{X}$ с поточечными операциями.

Рассмотрим вложение алгебры симметрических функций $\Lambda$ в алгебру $\operatorname{Fun}(\mathbb{K})$, определенное на образующих $p_{k}, k=1,2, \ldots$, следующим образом: $p_{k} \rightarrow p_{k}(\lambda)$ $:=\sum_{i=1}^{\ell(\lambda)} \lambda_{i}^{k}$. Таким образом, каждому элементу $f \in \Lambda$ соответствует функция из $\operatorname{Fun}(\mathbb{K})$, которую мы обозначим через $f(\lambda)$.

Для $f \in \Lambda$ обозначим через $f_{n}$ ограничение функции $f(\cdot) \in \mathrm{Fun}(\mathbb{K})$ на $\mathbb{K}_{n} \subset \mathbb{K}$. Легко проверить, что подалгебра $\Lambda \subset \operatorname{Fun}(\mathbb{K})$ разделяет точки, а следовательно, функции вида $f_{n}, f \in \Lambda$, исчерпывают (конечномерное) пространство $\operatorname{Fun}\left(\mathbb{K}_{n}\right)$.

Цель этого параграфа - доказать следующее предложение, которое будет необходимо для доказательства леммы 4.1 ниже.

Предложение 3.1. Рассмотрим переходный оператор $n$-й марковской иепи вверx/вниз $T_{n}: \operatorname{Fun}\left(\mathbb{K}_{n}\right) \rightarrow \operatorname{Fun}\left(\mathbb{K}_{n}\right)$, соответствующий двухпараметрической структуре разбиений Ювенса-Питмана. Его действие на функиии $\mathbf{m}_{\mu}^{*}, \mu \in \mathbb{K}$, имеет ви $\left.\partial^{1}\right)$

$$
\begin{aligned}
\left(T_{n}-\mathbf{1}\right)\left(\mathbf{m}_{\mu}^{*}\right)_{n}=- & \frac{|\mu|(|\mu|-1+\theta)}{(n+1)(n+\theta)}\left(\mathbf{m}_{\mu}^{*}\right)_{n} \\
& +\frac{n+1-|\mu|}{(n+1)(n+\theta)} \sum_{\substack{i=1 \\
\mu_{i} \geqslant 2}}^{\ell(\mu)} \mu_{i}\left(\mu_{i}-1-\alpha\right)\left(\mathbf{m}_{\mu-\square\left(\mu_{i}\right)}^{*}\right)_{n} \\
& +\frac{n+1-|\mu|}{(n+1)(n+\theta)}[\mu: 1](\theta+\alpha(\ell(\mu)-1))\left(\mathbf{m}_{\mu-\square(1)}^{*}\right)_{n} .
\end{aligned}
$$

\footnotetext{
1) Здесь и далее 1 означает тождественный оператор.
} 
Замечание 3.2. Предложение утверждает, что $\left(T_{n}-\mathbf{1}\right)\left(\mathbf{m}_{\mu}^{*}\right)_{n}$ представляет собой линейную комбинацию функции $\left(\mathbf{m}_{\mu}^{*}\right)_{n}$ (первое слагаемое правой части) и функций вида $\left(\mathbf{m}_{\varkappa}^{*}\right)_{n}$ по всем $\varkappa$, таким, что $\varkappa \nearrow \mu$. Сумма по $i$ в правой части описывает $\varkappa$ той же длины, что и $\mu$. Заметим, что она содержит повторяющиеся слагаемые, если $\mu$ имеет одинаковые строки длины $\geqslant 2$. Последнее слагаемое отлично от нуля, если $[\mu: 1]>0$, и в этом случае соответствует диаграмме $\varkappa=\mu-\square(1)$, полученной из $\mu$ выкидыванием строки длины 1.

Приведем две вспомогательные формулы, касающиеся факториальных функций и чисел путей $g(\mu, \lambda)$, определенных в разд. 1.1. Эти формулы используются ниже для доказательства предложения 3.1. Пусть $|\lambda|=n \geqslant m=|\mu|, \lambda, \mu \in \mathbb{K}$. Тогда

$$
\frac{g(\mu, \lambda)}{g(\lambda)}=\frac{\left(m_{\mu}^{*}\right)_{n}\left(\lambda_{1}, \ldots, \lambda_{\ell(\lambda)}\right)}{n(n-1) \cdots(n-m+1)}
$$

и

$$
g(\mu, \lambda)=\sum_{\varkappa: \varkappa / \lambda} \kappa(\varkappa, \lambda) g(\mu, \varkappa)
$$

Формула (6) может быть проверена непосредственно, а (7) вытекает из определения чисел $g(\mu, \lambda)$ и $\kappa(\varkappa, \lambda)$, см. разд. 1.1.

Для доказательства предложения 3.1 представим оператор $T_{n}$ в виде композиции операторов «вниз» $D_{n+1, n}: \operatorname{Fun}\left(\mathbb{K}_{n}\right) \rightarrow \operatorname{Fun}\left(\mathbb{K}_{n+1}\right)$ и «вверх» $U_{n, n+1}$ : $\operatorname{Fun}\left(\mathbb{K}_{n+1}\right) \rightarrow \operatorname{Fun}\left(\mathbb{K}_{n}\right)$, действующих на функции.

Оператор $D_{n+1, n}$ строится по переходным вероятностям вниз и не зависит от параметров $\alpha$ и $\theta$, в то время как оператор $U_{n, n+1}$ строится по переходным вероятностям вверх и от параметров зависит:

$$
\begin{aligned}
\left(D_{n+1, n} f_{n}\right)(\lambda) & :=\sum_{\mu: \mu \nearrow \lambda} p^{\downarrow}(\lambda, \mu) f_{n}(\mu), & & \lambda \in \mathbb{K}_{n+1}, \\
\left(U_{n, n+1} f_{n+1}\right)(\mu) & :=\sum_{\lambda: \lambda \backslash \mu} p^{\uparrow}(\mu, \lambda) f_{n+1}(\lambda), & & \mu \in \mathbb{K}_{n} .
\end{aligned}
$$

Эти операторы являются сопряженными к соответствующим операторам, действующим на меры. Последние операторы действуют в соответствии со своими названиями, например, $D_{n+1, n}^{*}: \mathscr{M}\left(\mathbb{K}_{n+1}\right) \rightarrow \mathscr{M}\left(\mathbb{K}_{n}\right)$, где $\mathscr{M}(\mathscr{X})$ - меры на пространстве $\mathscr{X}$.

Из определения $n$-й марковской цепи вверх/вниз (разд. 1.2) следует, что $T_{n}=U_{n, n+1} \circ D_{n+1, n}: \operatorname{Fun}\left(\mathbb{K}_{n}\right) \rightarrow \operatorname{Fun}\left(\mathbb{K}_{n}\right), n \in \mathbb{Z}_{>0}$. Предложение 3.1 является простым следствием такой леммы:

Лемма 3.3. (1) Существует единственный оператор $\widetilde{D}: \Lambda \rightarrow \Lambda$, такой, что для всех $n \in \mathbb{Z}_{\geqslant 0}$ и $f \in \Lambda$ выполняется равенство

$$
D_{n+1, n} f_{n}=\frac{1}{n+1}(\widetilde{D} f)_{n+1} .
$$

В базисе $\left\{\mathbf{m}_{\mu}^{*}\right\}_{\mu \in \mathbb{K}}$ алгебры $\Lambda$ этот оператор имеет вид

$$
\widetilde{D} \mathbf{m}_{\mu}^{*}=\left(p_{1}-|\mu|\right) \mathbf{m}_{\mu}^{*} .
$$


(2) Существует единственный оператор $\widetilde{U}: \Lambda \rightarrow \Lambda$, такой, что для всех $n \in \mathbb{Z}_{\geqslant 0} \quad u h \in \Lambda$

$$
U_{n, n+1} h_{n+1}=\frac{1}{n+\theta}(\widetilde{U} h)_{n} .
$$

В базисе $\left\{\mathbf{m}_{\mu}^{*}\right\}_{\mu \in \mathbb{K}}$ алгебры $\Lambda$ этот оператор имеет вид

$$
\begin{aligned}
\widetilde{U} \mathbf{m}_{\mu}^{*}=\left(p_{1}+\theta+|\mu|\right) \mathbf{m}_{\mu}^{*}+ & \sum_{\substack{i=1 \\
\mu_{i} \geqslant 2}}^{\ell(\mu)} \mu_{i}\left(\mu_{i}-1-\alpha\right) \mathbf{m}_{\mu-\square\left(\mu_{i}\right)}^{*}+[\mu: 1](\theta+\alpha(\ell(\mu)-1)) \mathbf{m}_{\mu-\square(1)}^{*} .
\end{aligned}
$$

Отметим, что в правой части формулы (10) стоит сумма, аналогичная той, которая поясняется в замечании 3.2.

Доказательство. (1) Будем использовать связь (6) факториальных функций с числами путей $g(\mu, \lambda)$ и рекуррентные соотношения (7) для последних.

Пусть $\mu \in \mathbb{K}, \nu \in \mathbb{K}_{n+1}$. Мы предполагаем, что $n \geqslant|\mu|$, в противном случае $\left(m_{\mu}^{*}\right)_{n}=0$. Имеем

$$
\begin{aligned}
\left(D_{n+1, n}\left(m_{\mu}^{*}\right)_{n}\right)(\nu) & =\sum_{\lambda: \lambda \nearrow \nu} p^{\downarrow}(\nu, \lambda)\left(m_{\mu}^{*}\right)_{n}(\lambda)=\sum_{\lambda: \lambda \nearrow \nu} \frac{g(\lambda)}{g(\nu)} \kappa(\lambda, \nu)\left(m_{\mu}^{*}\right)_{n}(\lambda) \\
& =\sum_{\lambda: \lambda \nearrow \nu} \frac{g(\lambda)}{g(\nu)} \kappa(\lambda, \nu) n^{\downarrow|\mu|} \frac{g(\mu, \lambda)}{g(\lambda)}=\frac{n^{\downarrow|\mu|}}{g(\nu)} \sum_{\lambda: \lambda \nearrow \nu} \kappa(\lambda, \nu) g(\mu, \lambda) \\
& =n^{\downarrow|\mu|} \frac{g(\mu, \nu)}{g(\nu)}=\frac{n+1-|\mu|}{n+1}\left(m_{\mu}^{*}\right)_{n+1}(\nu) .
\end{aligned}
$$

Таким образом, для всех $\mu \in \mathbb{K}$

$$
D_{n+1, n}\left(m_{\mu}^{*}\right)_{n}=\frac{1}{n+1}\left(\left(p_{1}-|\mu|\right) m_{\mu}^{*}\right)_{n+1},
$$

поскольку функция $p_{1}$ на $\mathbb{K}_{n+1}$ тождественно равна $n+1$. Значит, оператор $\widetilde{D}$ существует и в базисе $\left\{m_{\mu}^{*}\right\}_{\mu \in \mathbb{K}}$ имеет вид $\widetilde{D} m_{\mu}^{*}=\left(p_{1}-|\mu|\right) m_{\mu}^{*}$. Умножив обе части на $\prod_{k \geqslant 1}[\mu: k]$ !, получим искомое выражение $(9)$ для оператора $\widetilde{D}$ в базиce $\left\{\mathbf{m}_{\mu}^{*}\right\}_{\mu \in \mathbb{K}}$. Единственность оператора $\widetilde{D}$ следует из того, что $\Lambda$ инъективно отображается в $\operatorname{Fun}(\mathbb{K})$.

(2) Фиксируем $\mu \in \mathbb{K}$ и положим $l:=\ell(\mu)$. Пусть $\nu \in \mathbb{K}_{n}$. Есть явная формула

$$
\left(\mathbf{m}_{\mu}^{*}\right)_{n}(\nu)=\sum \nu_{j_{1}}^{\downarrow \mu_{1}} \cdots \nu_{j_{l}}^{\downarrow \mu_{l}}
$$

где суммирование ведется по всем попарно различным $j_{1}, \ldots, j_{l}$ от 1 до $\ell(\nu)$, и поэтому существует возможность доказать (10) непосредственно.

Из определения (8) оператора $U_{n, n+1}$ и вида переходных вероятностей вверх для структуры разбиений Ювенса-Питмана (разд. 1.3) имеем

$$
\begin{array}{r}
\left(U_{n, n+1}\left(\mathbf{m}_{\mu}^{*}\right)_{n+1}\right)(\nu)=\frac{1}{n+\theta}\left[\sum_{i=1}^{\ell(\nu)}\left(\nu_{i}-\alpha\right)\left(\mathbf{m}_{\mu}^{*}\right)_{n+1}\left(\nu+\widehat{\square}\left(\nu_{i}+1\right)\right)\right. \\
\left.+(\theta+\ell(\nu) \alpha)\left(\mathbf{m}_{\mu}^{*}\right)_{n+1}(\nu+\widehat{\square}(1))\right] .
\end{array}
$$


Преобразуем правую часть, чтобы получить (10).

1. Сначала отметим два простых свойства:

1a. $\quad(a+1)^{\downarrow b}-a^{\downarrow b}=b \cdot a^{\downarrow(b-1)} ; \quad$ 1b. $\quad a \cdot a^{\downarrow b}=a^{\downarrow(b+1)}+b \cdot a^{\downarrow b}$.

2. Для произвольных $c \in\{1, \ldots, l\}$ и $i \in\{1, \ldots, \ell(\nu)+1\}$ положим

$$
S_{c}(i):=\sum \nu_{j_{1}}^{\downarrow \mu_{1}} \cdots \nu_{j_{c-1}}^{\downarrow \mu_{c-1}} \nu_{j_{c+1}}^{\downarrow \mu_{c+1}} \cdots \nu_{j_{l}}^{\downarrow \mu_{j_{l}}},
$$

где суммирование ведется по всем попарно различным $j_{1}, \ldots, j_{c-1}, j_{c+1}, \ldots, j_{l}$, которые пробегают множество $\{1, \ldots, i-1, i+1, \ldots, \ell(\nu)+1\}$.

3. Разберемся с отдельным слагаемым в правой части (11). Покажем, что

$$
\left(\mathbf{m}_{\mu}^{*}\right)_{n+1}(\nu+\widehat{\square}(1))=\left(\mathbf{m}_{\mu}^{*}\right)_{n}(\nu)+[\mu: 1]\left(\mathbf{m}_{\mu-\square(1)}^{*}\right)_{n}(\nu) .
$$

Ясно, что $\left(\mathbf{m}_{\mu}^{*}\right)_{n+1}(\nu+\widehat{\square}(1))=\sum(\nu+\widehat{\square}(1))_{j_{1}}^{\downarrow \mu_{1}} \cdots(\nu+\widehat{\square}(1))_{j_{l}}^{\downarrow \mu_{l}}$, где суммирование ведется по всем попарно различным наборам $j_{1}, \ldots, j_{l}$ от 1 до $\ell(\nu)+1$. Представим $\left(\mathbf{m}_{\mu}^{*}\right)_{n+1}(\nu+\widehat{\square}(1))$ в виде $\Sigma_{1}+\Sigma_{2}$, где в $\Sigma_{1}$ входят слагаемые, для которых $j_{1}, \ldots, j_{l} \leqslant \ell(\nu)$, а в $\Sigma_{2}-$ (оставшиеся) слагаемые, для которых существует число $c \in\{1, \ldots, l\}$, определяемое условием $j_{c}=\ell(\nu)+1$. Легко видеть, что $\Sigma_{1}=\left(\mathbf{m}_{\mu}^{*}\right)_{n}(\nu)$. Запишем $\Sigma_{2}$, группируя слагаемые с одинаковыми $c$ :

$$
\Sigma_{2}=\sum_{c=1}^{l} 1^{\downarrow \mu_{c}} \cdot S_{c}(\ell(\nu)+1)=[\mu: 1]\left(\mathbf{m}_{\mu-\square(1)}^{*}\right)_{n}(\nu) .
$$

Первое равенство следует из того, что $(\nu+\widehat{\square}(1))_{j_{c}}^{\downarrow \mu_{c}}=1^{\downarrow \mu_{c}}$ и $(\nu+\widehat{\square}(1))_{j_{k}}=\nu_{j_{k}}$ для $k \neq c$, а второе - из того, что $1^{\downarrow b}=0$, если $b \geqslant 2$, и $1^{\downarrow 1}=1$. Таким образом, (12) установлено.

4. Теперь разберемся с суммой по $i$ в правой части (11).

Сначала зафиксируем $i$ от 1 до $\ell(\nu)$. Аналогично п. 3 имеем

$$
\begin{gathered}
\left(\mathbf{m}_{\mu}^{*}\right)_{n}(\nu)=\sum_{c=1}^{l} \nu_{i}^{\downarrow \mu_{c}} S_{c}(i)+\sum_{j_{1}, \ldots, j_{l} \neq i} \nu_{j_{1}}^{\downarrow \mu_{1}} \cdots \nu_{j_{l}}^{\downarrow \mu_{l}}, \\
\left(\mathbf{m}_{\mu}^{*}\right)_{n+1}\left(\nu+\widehat{\square}\left(\nu_{i}+1\right)\right)=\sum_{c=1}^{l}\left(\nu_{i}+1\right)^{\downarrow \mu_{c}} S_{c}(i)+\sum_{j_{1}, \ldots, j_{l} \neq i} \nu_{j_{1}}^{\downarrow \mu_{1}} \cdots \nu_{j_{l}}^{\downarrow \mu_{l}} .
\end{gathered}
$$

Используя п. 1а, получаем

$$
\left(\mathbf{m}_{\mu}^{*}\right)_{n+1}\left(\nu+\widehat{\square}\left(\nu_{i}+1\right)\right)-\left(\mathbf{m}_{\mu}^{*}\right)_{n}(\nu)=\sum_{c=1}^{l} \mu_{c} \nu_{i}^{\downarrow\left(\mu_{c}-1\right)} S_{c}(i) .
$$

Таким образом, сумма по $i$ в правой части формулы (11) равна

$$
\begin{aligned}
\sum_{i=1}^{l}\left(\nu_{i}-\alpha\right)\left(\mathbf{m}_{\mu}^{*}\right)_{n+1}\left(\nu+\widehat{\square}\left(\nu_{i}+1\right)\right) & \\
=(|\nu|-\ell(\nu) \alpha)\left(\mathbf{m}_{\mu}^{*}\right)_{n}(\nu)+\sum_{i=1}^{\ell(\nu)} \sum_{c=1}^{l}\left(\nu_{i}-\alpha\right) \mu_{c} \nu_{i}^{\downarrow}\left(\mu_{c}-1\right) & S_{c}(i) .
\end{aligned}
$$


Фиксируем $c$ от 1 до $l$. Используя п. $1 \mathrm{~b}$, получим

$$
\begin{aligned}
& \sum_{i=1}^{\ell(\nu)}\left(\nu_{i}-\alpha\right) \mu_{c} \nu_{i}^{\downarrow\left(\mu_{c}-1\right)} S_{c}(i) \\
& =S_{c}(i)\left[\mu_{c} \sum_{i=1}^{\ell(\nu)} \nu_{i}^{\downarrow} \mu_{c}+\mu_{c}\left(\mu_{c}-1\right) \sum_{i=1}^{\ell(\nu)} \nu_{i}^{\downarrow}\left(\mu_{c}-1\right)-\alpha \mu_{c} \sum_{i=1}^{\ell(\nu)} \nu_{i}^{\downarrow}\left(\mu_{c}-1\right)\right] \\
& = \begin{cases}\mu_{c}\left(\mathbf{m}_{\mu}^{*}\right)_{n}(\nu)+\mu_{c}\left(\mu_{c}-1-\alpha\right)\left(\mathbf{m}_{\mu-\square\left(\mu_{c}\right)}\right)_{n}(\nu), & \text { если } \mu_{c} \geqslant 2, \\
\left(\mathbf{m}_{\mu}^{*}\right)_{n}(\nu)-\alpha(\ell(\nu)-(l-1))\left(\mathbf{m}_{\mu-\square(1)}^{*}\right)_{n}(\nu), & \text { если } \mu_{c}=1 .\end{cases}
\end{aligned}
$$

5. Комбинируя пп. 3 и 4 и вспоминая, что $|\nu|=n$ и $\ell(\mu)=l$, получаем

$$
\begin{aligned}
& \left(U_{n, n+1}\left(\mathbf{m}_{\mu}^{*}\right)_{n+1}\right)(\nu) \\
& =\left(\mathbf{m}_{\mu}^{*}\right)_{n}(\nu)+\frac{1}{p_{1}(\nu)+\theta}\left[|\mu|\left(\mathbf{m}_{\mu}^{*}\right)_{n}(\nu)+\sum_{\substack{c=1 \\
\mu_{c} \geqslant 2}}^{\ell(\mu)} \mu_{c}\left(\mu_{c}-1-\alpha\right)\left(\mathbf{m}_{\mu-\square\left(\mu_{c}\right)}^{*}\right)_{n}(\nu)\right. \\
& \left.+[\mu: 1](\theta+\alpha(\ell(\mu)-1))\left(\mathbf{m}_{\mu-\square(1)}^{*}\right)_{n}(\nu)\right] .
\end{aligned}
$$

Это совпадает с искомым выражением (10). Единственность оператора $\widetilde{U}$ вновь следует из того, что $\Lambda \rightarrow \operatorname{Fun}(\mathbb{K})$ есть вложение.

\section{§4. Сходимость марковских цепей вверх/вниз}

В этом параграфе рассматривается предел марковских цепей $T_{n}$ на $\mathbb{K}_{n}$ при $n \rightarrow \infty$ в случае, когда параметры $\alpha$ и $\theta$ лежат в основной серии. Этот предел является марковским процессом на $\bar{\nabla}_{\infty}$ с непрерывным временем и обозначается через $\mathbf{X}_{\alpha, \theta}(t)$.

Доказательства в этом параграфе используют результаты книги [5]. Применение этих результатов базируется на алгебраических вычислениях §3 и аналогично рассуждениям в [2, §1]. В силу указанной аналогии доказательства, которые повторяют работу [2], не приводятся.

4.1. Построение процесса $\mathrm{X}_{\boldsymbol{\alpha}, \boldsymbol{\theta}}(\boldsymbol{t})$. Сначала приведем некоторые необходимые обозначения.

Каждый оператор $T_{n}$ действует в конечномерном пространстве функций $\operatorname{Fun}\left(\mathbb{K}_{n}\right), n=1,2, \ldots$. Эти пространства можно считать банаховыми с супремум-нормой $\|\cdot\|_{n}$. В разд. 2.3 были введены вложения $\iota_{n}: \mathbb{K}_{n} \hookrightarrow \bar{\nabla}_{\infty}$. Пусть $\pi_{n}$ - соответствующие им проекции пространств функций:

$$
\left(\pi_{n}(f)\right)(\lambda):=f\left(\iota_{n}(\lambda)\right), \quad \lambda \in \mathbb{K}_{n}, f \in C\left(\bar{\nabla}_{\infty}\right) .
$$

В $\S 2$ была введена плотная подалгебра $\mathscr{F} \subset C\left(\bar{\nabla}_{\infty}\right)$. Алгебра $\mathscr{F}$ допускает возрастающую фильтрацию конечномерными пространствами

$$
\mathscr{F}^{0} \subset \mathscr{F}^{1} \subset \mathscr{F}^{2} \cdots \subset \mathscr{F}, \quad \bigcup_{m=0}^{\infty} \mathscr{F}^{m}=\mathscr{F} .
$$

Проще всего определить эту фильтрацию как образ фильтрации алгебры $\Lambda$ по степеням полиномов (от формальных переменных $y_{i}$, см. разд. 2.1) при факторизации, поскольку $\mathscr{F} \cong \Lambda^{\circ}=\Lambda /\left(p_{1}-1\right) \Lambda$. 
Из замечания 2.1 и того, что $\operatorname{dim} \mathscr{F}^{m}<\infty$, следует, что все проекции $\pi_{n}$ являются взаимно однозначными на $\mathscr{F}^{m}$ (при фиксированном $m$ ) для всех достаточно больших $n$.

Докажем, что генераторы марковских цепей вверх/вниз сходятся к некоторому оператору $A$ в пространстве $C\left(\bar{\nabla}_{\infty}\right)$.

Лемма 4.1. При фиксированном $m$ для всех достаточно больших $n$ каждое подпространство $\pi_{n}\left(\mathscr{F}^{m}\right)$ является инвариантным относительно оператора $T_{n}$. C точностью до отождествления $\mathscr{F}^{m}=\pi_{n}\left(\mathscr{F}^{m}\right)$ существует предел

$$
\lim _{n \rightarrow \infty} n^{2}\left(T_{n}-\mathbf{1}\right) f=A f \quad \text { для всех } f \in \mathscr{F}
$$

в каждом конечномерном пространстве $\mathscr{F}^{m}$. Таким образом, возникает оператор $A: \mathscr{F} \rightarrow \mathscr{F}$, для которого $A \mathscr{F}^{m} \subset \mathscr{F}^{m}, m \geqslant 0$. Действие этого оператора на функиии $\mathbf{m}_{\mu}^{\circ} \in \Lambda^{\circ} \cong \mathscr{F}, \mu \in \mathbb{K}$ (определенные в 2 ), имеет следующий явный вид:

$$
\begin{gathered}
A \mathbf{m}_{\mu}^{\circ}=-|\mu|(|\mu|-1+\theta) \mathbf{m}_{\mu}^{\circ}+\sum_{\substack{c=1 \\
\mu_{c} \geqslant 2}}^{\ell(\mu)} \mu_{c}\left(\mu_{c}-1-\alpha\right) \mathbf{m}_{\mu-\square\left(\mu_{c}\right)}^{\circ}+[\mu: 1](\theta+\alpha(\ell(\mu)-1)) \mathbf{m}_{\mu-\square(1)}^{\circ} .
\end{gathered}
$$

Заметим, что $A$ переводит функцию $\mathbf{m}_{\varnothing}^{\circ} \equiv 1$ в 0, как и должно быть.

Доказательство. Ясно, что для всех достаточно больших $n$ пространство $\pi_{n}\left(\mathscr{F}^{m}\right)$ является линейной оболочкой функций $\left(\mathbf{m}_{\mu}^{*}\right)_{n}$, где $|\mu| \leqslant m$. Таким образом, свойство инвариантности $\pi_{n}\left(\mathscr{F}^{m}\right)$ относительно $T_{n}$ следует из предложения 3.1 , так как видно, что оператор $T_{n}$ не повышает степень функций $\left(\mathbf{m}_{\mu}^{*}\right)_{n} \in \operatorname{Fun}\left(\mathbb{K}_{n}\right)$ (которая равна $\left.|\mu|\right)$.

Таким образом, отождествляя $\pi_{n}\left(\mathscr{F}^{m}\right)$ с $\mathscr{F}^{m}$ (что имеет смысл для достаточно больших $n$ при фиксированном $m$ ), мы можем говорить, что $\mathscr{F}^{m}$ инвариантны под действием $T_{n}$. Теперь можно одновременно провести доказательство формул (13) и (14).

Рассмотрим отображение $\Lambda \rightarrow \operatorname{Fun}\left(\mathbb{K}_{n}\right), f \mapsto f_{[n]}$, где

$$
f_{[n]}(\lambda):=\pi_{n}\left(f^{\circ}\right)(\lambda)=f^{\circ}\left(\frac{\lambda_{1}}{n}, \frac{\lambda_{2}}{n}, \ldots, \frac{\lambda_{\ell(\lambda)}}{n}, 0,0, \ldots\right), \quad \lambda \in \mathbb{K}_{n}, f \in \Lambda .
$$

Другими словами, функция $f_{[n]}$ на $\mathbb{K}_{n}$ определяется как ограничение непрерывной функции $f^{\circ} \in C\left(\bar{\nabla}_{\infty}\right)$ на $\iota_{n}\left(\mathbb{K}_{n}\right) \subset \bar{\nabla}_{\infty}$. Пусть $G_{s}: \Lambda \rightarrow \Lambda$ для любого $s>0$ - автоморфизм алгебры $\Lambda$, определяемый на ее базисе формулой

$$
G_{s} \mathbf{m}_{\mu}=s^{|\mu|} \mathbf{m}_{\mu}, \quad \mu \in \mathbb{K} .
$$

На однородной компоненте степени ${ }^{1)} k, k=0,1,2, \ldots$, этот автоморфизм сводится к умножению на число $s^{k}$.

Тогда мы имеем следующее выражение для $f_{n}$, где $f \in \Lambda$ :

$$
f_{n}(\lambda)=\left(G_{n} f\right)_{[n]}(\lambda), \quad \lambda \in \mathbb{K}_{n} .
$$

1) Однородная компонента степени $k$ в алгебре $\Lambda$ состоит из всех однородных симметрических функций степени $k$ и является линейной оболочкой элементов $\mathbf{m}_{\mu},|\mu|=k$. 
Действительно, так как $\iota_{n}\left(\mathbb{K}_{n}\right) \subset \nabla_{\infty}$, то для всех $\varphi \in \Lambda$ и $\lambda \in \mathbb{K}_{n}$ значение $\varphi^{\circ}\left(\iota_{n}(\lambda)\right)=\varphi_{[n]}(\lambda)$ совпадает с результатом прямой подстановки координат точки $\iota_{n}(\lambda)=\left(\lambda_{1} / n, \ldots, \lambda_{\ell(\lambda)} / n, 0,0, \ldots\right) \in \nabla_{\infty}$ в элемент $\varphi \in \Lambda$ (см. замечание 5.2 ниже). В $\S 2$ было отмечено, что однородная компонента наибольшей степени в $\mathbf{m}_{\mu}^{*}$ есть $\mathbf{m}_{\mu}$. Поэтому для всех $\mu \in \mathbb{K}$

$$
\lim _{n \rightarrow \infty} n^{-|\mu|} G_{n} \mathbf{m}_{\mu}^{*}=\mathbf{m}_{\mu}
$$

Подставим выражение $(15)$ для $f_{n}(\lambda)$ в формулу для $\left(T_{n}-\mathbf{1}\right)\left(\mathbf{m}_{\mu}^{*}\right)_{n}$ из предложения 3.1, домножим результат на $n^{-|\mu|}$ и перейдем к пределу по $n$. Мы получим сходимость (13) операторов $n^{2}\left(T_{n}-\mathbf{1}\right) \mathrm{k}$ оператору $A: \mathscr{F} \rightarrow \mathscr{F}$, заданному формулой $(14)$, в каждом конечномерном подпространстве ${ }^{1)} \mathscr{F}^{m}$, что и требовалось.

Заметим, что набор элементов $\mathbf{m}_{\mu}^{\circ}, \mu \in \mathbb{K}$, пространства $\mathscr{F}$ не является линейно независимым. Поэтому неочевидно, что (14) корректно задает $A$. Корректность следует из доказательства предыдущей леммы: из сходимости (13) вытекает существование оператора $A: \mathscr{F} \rightarrow \mathscr{F}$, действие которого на функции $\mathbf{m}_{\mu}^{\circ}$ задается формулой (14). Так как семейство $\mathbf{m}_{\mu}^{\circ}, \mu \in \mathbb{K}$, содержит базис пространства $\mathscr{F},(14)$ в самом деле корректно задает оператор в $\mathscr{F}$.

Теперь перейдем к сходимости дискретных полугрупп $\left\{\mathbf{1}, T_{n}, T_{n}^{2}, \ldots\right\}$ к непрерывной консервативной марковской полугруппе $\{T(t)\}_{t \geqslant 0}$ в банаховом пространстве $C\left(\bar{\nabla}_{\infty}\right)$, которая строится по оператору $A$. Напомним, что консервативной марковской полугруппой называется сильно непрерывная группа сжимающих операторов в $C\left(\bar{\nabla}_{\infty}\right)$, каждый из которых сохраняет положительные функции и константу 1.

Чтобы придать строгий смысл сходимости дискретных полугрупп к непрерывной, воспользуемся определением из [5, гл. 1, разд. 6]:

Определение 4.2. Будем говорить, что некая последовательность функций $\left\{f_{n} \in \operatorname{Fun}\left(\mathbb{K}_{n}\right)\right\}$ сходится к функции $f \in C\left(\bar{\nabla}_{\infty}\right)$, если $\left\|f_{n}-\pi_{n}(f)\right\|_{n} \rightarrow 0$ при $n \rightarrow \infty$. Здесь $\|\cdot\|_{n}-$ супремум-норма пространства $\operatorname{Fun}\left(\mathbb{K}_{n}\right)$. В этом случае будем писать $f_{n} \rightarrow f$.

Для сходимости полугрупп $\left\{\mathbf{1}, T_{n}, T_{n}^{2}, \ldots\right\}$ к $\{T(t)\}_{t \geqslant 0}$ необходимо сделать такое масштабное преобразование времени, чтобы один шаг $n$-й цепи вверх/вниз соответствовал малому интервалу времени порядка $n^{-2}$.

Предложение 4.3. (1) Oператор $A: \mathscr{F} \rightarrow \mathscr{F}$, определенный в лемме 4.1, является замыкаемым в пространстве $C\left(\bar{\nabla}_{\infty}\right)$.

(2) Замыкание $\bar{A}$ оператора $A$ порождает консервативную марковскую полугрупny $\{T(t)\}_{t \geqslant 0}$ в $C\left(\bar{\nabla}_{\infty}\right)$.

(3) Дискретные полугруппь $\left\{\mathbf{1}, T_{n}, T_{n}^{2}, \ldots\right\}$ при $n \rightarrow \infty$ сходятся $\kappa$ полуzрynпе $\{T(t)\}_{t \geqslant 0}$ в том смысле, что

$$
T_{n}^{\left[n^{2} t\right]} \pi_{n}(f) \rightarrow T(t) f \quad \text { для всех } f \in \Lambda
$$

(предел понимается в смысле определения 4.2) для всех $t \geqslant 0$ равномерно на ограниченных интервалах.

1) Заметим, что операторы $n^{2}\left(T_{n}-\mathbf{1}\right)$ могут быть определены на $\mathscr{F} m$ (при фиксированном $m$, начиная с некоторого $n)$ с использованием отождествления $\pi_{n}\left(\mathscr{F}^{m}\right)$ с $\mathscr{F}^{m}$. 
Предложение доказывается аналогично предложению 1.4 из [2], оно следует из сходимости генераторов (лемма 4.1). В доказательстве используется теорема 6.5 из [5, гл. 1], а также лемма 2.11 из [5, гл. 1].

Далее, из [5, гл. 4, теорема 2.7] сразу следует, что $\{T(t)\}_{t \geqslant 0}$ является полугруппой строго марковского семейства с траекториями, непрерывными справа и имеющими пределы слева для всех $t \geqslant 0$.

Оператор $A$ будем называть предгенератором полугруппы $\{T(t)\}_{t \geqslant 0}$ и процесса $\mathbf{X}_{\alpha, \theta}(t)$. Таким образом, для каждой пары параметров $\alpha, \theta$ из основной серии построен марковский процесс $\mathbf{X}_{\alpha, \theta}(t)$ на $\bar{\nabla}_{\infty}$, который может начинаться из любой точки и с любого начального вероятностного распределения.

4.2. Некоторые свойства процесса $\mathbf{X}_{\alpha, \theta}(t)$. В этом разделе формулируются и поясняются свойства процесса $\mathbf{X}_{\alpha, \theta}(t)$, которые аналогичны приведенным в работе [2].

Сначала сформулируем свойства этого процесса, которые являются прямыми следствиями его конструкции как предела конечных марковских цепей $T_{n}$, сохраняющих меры $M_{n}$ на $\mathbb{K}_{n}$. Для их доказательства используется сходимость мер $M_{n}$ (теорема Кингмана, разд. 2.3) и свойства цепей вверх/вниз (разд. 1.2).

Инвариантная мера (ср. [2, предложение 1.6]). Распределение ПуассонаДирихле $\mathrm{PD}(\alpha, \theta)$ является инвариантной мерой для прочесса $\mathbf{X}_{\alpha, \theta}(t)$.

Это следует из того, что каждая цепь $T_{n}$ сохраняет меру $M_{n}$.

Обратимость процесса (ср. [2, предложение 1.7 и теорема 7.3 (2)]). Процесс $\mathbf{X}_{\alpha, \theta}(t)$ является обратимым относительно меры $\mathrm{PD}(\alpha, \theta)$.

Это следует из того, что каждая цепь $T_{n}$ обратима относительно $M_{n}$.

Сходимость конечномерных распределений (ср. [2, предложение 1.8]). Будем считать, что $\mathbf{X}_{\alpha, \theta}(t)$ и все цепи $T_{n}$ начинаются с инвариантного распределения. Тогда все конечномерные распределения чепей $T_{n}$ сходлтся к конечномерным распределениям процесса $\mathbf{X}_{\alpha, \theta}(t)$ nри $n \rightarrow \infty$, если сделать масштабное преобразование времени, описанное перед предложением 4.3.

Теперь приведем свойства, которые вытекают из представления предгенератора $A$ процесса $\mathbf{X}_{\alpha, \theta}$ в виде (14).

Описание спектра марковского генератора в $L^{2}\left(\bar{\nabla}_{\infty}, \operatorname{PD}(\alpha, \theta)\right)$. Предгенератор А действует в пространстве $\mathscr{F} \cong \Lambda^{\circ}$. Оно разлагается в ортогональную (по отношению $к$ скалярному произведению в $\Lambda^{\circ}$, унаследованному из $\left.L^{2}\left(\bar{\nabla}_{\infty}, \mathrm{PD}(\alpha, \theta)\right)\right)$ прямую сумму собственных подпространств оператора $A$. Спектр оператора A имеет вид

$$
\{0\} \cup\left\{-\sigma_{m}: m=2,3, \ldots\right\}, \quad \sigma_{m}=m(m-1+\theta) .
$$

Собственное значение 0 является простым, а кратность каждого $-\sigma_{m}$ равна количеству разбиений числа $m$ в сумму натуральных слагаемых, больших единиць.

Существование разложения пространства $\Lambda^{\circ}$ следует из того, что предгенератор $A$ симметричен (см. обратимость процесса выше) и сохраняет фильтрацию $\left\{\mathscr{F}^{m}\right\}$ пространства $\mathscr{F} \cong \Lambda^{\circ}$ (см. (14)). Факты о структуре спектра вытекают из того, что оператор $A$ является треугольным в базисе $\left\{\mathbf{m}_{\mu}^{\circ}\right\}_{\mu \in \mathbb{K},[\mu: 1]=0}$, согласованном с фильтрацией $\left\{\mathscr{F}^{m}\right\}$.

Единственность инвариантной меры. Мера $\mathrm{PD}(\alpha, \theta)$ является единственной инвариантной мерой для прочесса $\mathbf{X}_{\alpha, \theta}(t)$.

Это доказывается аналогично теореме 7.3 (1) из [2]. 
Эргодичность. Процесс $\mathbf{X}_{\alpha, \theta}(t)$ является эргодическим относительно меpъь $\operatorname{PD}(\alpha, \theta)$.

Это утверждение следует из существования спектральной щели у генератора процесса $\mathbf{X}_{\alpha, \theta}(t)$, см. выше структуру спектра. Детальное доказательство дано в [2, теорема $7.3(3)]$.

\section{§5. Предгенератор $A$ процесса $\mathrm{X}_{\alpha, \theta}(t)$ как дифференциальный оператор}

Данный параграф посвящен более подробному изучению свойств предгенератора $A: \mathscr{F} \rightarrow \mathscr{F}$, который определяется в лемме 4.1. В разд. 5.1 и разд. 5.2 предполагается, что параметры $\alpha$ и $\theta$ лежат в основной серии. В разд. 5.3 рассматриваются вырожденные значения параметров.

5.1. Предгенератор $\boldsymbol{A}$ в естественных координатах. Напомним, что процесс $\mathbf{X}_{\alpha, \theta}(t)$ на $\bar{\nabla}_{\infty}$ имеет генератор $\bar{A}$, являющийся замыканием предгенератора $A: \mathscr{F} \rightarrow \mathscr{F}$. Алгебра $\mathscr{F} \cong \Lambda^{\circ}$ определяется в $\S 2$, а оператор $A$ задается

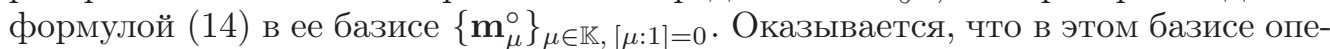
ратор $A$ может быть записан как дифференциальный оператор второго порядка в естественных координатах $x_{1}, x_{2}, \ldots$ на $\bar{\nabla}_{\infty}$. Сначала представим $\mathbf{m}_{\mu}^{\circ}(x)$ в этих координатах.

Предложение 5.1. Для всех $\mu \in \mathbb{K},[\mu: 1]=0$, бункиия $\mathbf{m}_{\mu}^{\circ}(x)$ на $\bar{\nabla}_{\infty}$ может быть записана в виде

$$
\mathbf{m}_{\mu}^{\circ}(x)=\sum_{i_{1}, \ldots, i_{\ell(\mu)}} x_{i_{1}}^{\mu_{1}} \cdots x_{i_{\ell(\mu)}}^{\mu_{\ell(\mu)}}, \quad x=\left(x_{1}, x_{2}, \ldots\right) \in \bar{\nabla}_{\infty},
$$

где суммирование ведется по всем попарно различным индексам $i_{1}, \ldots, i_{\ell(\mu)}$ от единицы до бесконечности.

Отметим, что если $[\mu: 1]>0$ и $\sum_{i=1}^{\infty} x_{i}<1$, то это утверждение неверно.

Доказательство. Предложение является частным случаем следующего более общего результата (принадлежащего Керову [15]), который позволяет вычислить любую функцию $\mathbf{m}_{\lambda}^{\circ}, \lambda \in \mathbb{K}$, в любой точке $x \in \bar{\nabla}_{\infty}$.

Пусть $f\left(x_{1}, x_{2}, \ldots\right)$ для $f \in \Lambda$ означает результат формальной подстановки координат точки $x$ в функцию $f$. Пусть $\lambda \in \mathbb{K},[\lambda: 1]=r \geqslant 0$. Тогда [15]

$$
\mathbf{m}_{\lambda}^{\circ}(x)=\sum_{k=0}^{r}\left(\begin{array}{l}
r \\
k
\end{array}\right)(\gamma(x))^{k} \mathbf{m}_{\lambda-k \cdot \square(1)}\left(x_{1}, x_{2}, \ldots\right), \quad x \in \bar{\nabla}_{\infty} .
$$

Здесь $\mathbf{m}_{\lambda}^{\circ}(x)$ - значение функции $\mathbf{m}_{\lambda}^{\circ}$ в точке $x$, построенное в $\S 2$ путем факторизации алгебры $\Lambda, \gamma(x)=1-\sum_{i=1}^{\infty} x_{i}$ и $\lambda-k \cdot \square(1)$ - диаграмма, полученная из $\lambda$ выкидыванием $k$ строк длины 1 .

Замечание 5.2. Из доказательства следует, что для любой функции $\varphi$ из нефакторизованной алгебры $\Lambda$ функция $\varphi^{\circ}(x)$ на $\bar{\nabla}_{\infty}$ может быть построена не только путем факторизации алгебры $\Lambda$, как объяснено в $\S 2$, но и непосредственно. А именно, для каждой точки $x \in \nabla_{\infty}\left(\right.$ где $\left.\sum_{i=1}^{\infty} x_{i}=1\right)$ положим $\varphi^{\circ}(x)$ равным результату формальной подстановки координат точки $x=\left(x_{1}, x_{2}, \ldots\right)$ в симметрическую функцию $\varphi \in \Lambda$. На весь симплекс $\bar{\nabla}_{\infty}$ функция $\varphi^{\circ}(x)$ продолжается по непрерывности. Этот способ подсказан комментарием после формулы (2.10) в [4]. 
Теперь нетрудно вычислить предгенератор $A$ в естественных координатах. Пусть $D$ обозначает следующее формальное выражение:

$$
D=\sum_{i=1}^{\infty} x_{i} \frac{\partial^{2}}{\partial x_{i}^{2}}-\sum_{i, j=1}^{\infty} x_{i} x_{j} \frac{\partial^{2}}{\partial x_{i} \partial x_{j}}-\sum_{i=1}^{\infty}\left(\theta x_{i}+\alpha\right) \frac{\partial}{\partial x_{i}} .
$$

Легко проверить, используя предложение 5.1 и (14), что $D \mathbf{m}_{\mu}^{\circ}=A \mathbf{m}_{\mu}^{\circ}$ для всех $\mu \in \mathbb{K}$ с условием $[\mu: 1]=0$. Поэтому $D f=A f$ для всех $f \in \mathscr{F} \subset C\left(\bar{\nabla}_{\infty}\right)$, и, таким образом, формула (3) из введения установлена.

Замечание 5.3. Правую часть формулы (16) можно понимать следующими двумя эквивалентными способами. Пусть $f \in \mathscr{F}$ и требуется вычислить $D f$. Тогда можно либо

(1) разложить $f$ по базису $\left\{\mathbf{m}_{\mu}^{\circ}\right\}_{\mu \in \mathbb{K},[\mu: 1]=0}$ алгебры $\mathscr{F}$ и применить оператор $D=A$ отдельно к каждому $\mathbf{m}_{\mu}^{\circ}$, либо

(2) вычислить $A f(x)$ сначала для $x \in \nabla_{\infty}$ путем прямого применения правой части формулы (16), а затем продолжить $A f(x)$ на $x \in \bar{\nabla}_{\infty}$ по непрерывности (ср. этот способ с замечанием 5.2).

Замечание 5.4. Существует соблазн применять $D$ (правую часть формулы $(16))$ к функциям $f$, не лежащим в $\mathscr{F}$. Однако если для некоторой функции $f \in C\left(\bar{\nabla}_{\infty}\right)$ выражение $D f$ имеет смысл, не очевидно, что $f$ обязана лежать в области определения генератора $\bar{A}$, и совсем не очевидно, что выражение $D f$ должно совпадать с $\bar{A} f$.

Рассмотрим пример $f=x_{1}+\alpha / \theta, \theta \neq 0$. Ясно, что $D f=-\theta f$. Если бы выполнялось равенство $D f=\bar{A} f$, то $f$ была бы собственной функцией генератора $\bar{A}$ с собственным значением $-\theta$. Поскольку $f \in L^{2}\left(\bar{\nabla}_{\infty}, \operatorname{PD}(\alpha, \theta)\right)$ и $-\theta$ не является собственным значением оператора $\bar{A}$ в этом пространстве (см. разд. $4.2)$, получаем противоречие.

В работе [27] показано, что при $\alpha=0$ функция $f=x_{1}$ лежит в области определения формы Дирихле, соответствующей оператору $\bar{A}$, а стало быть, в области определения оператора $\bar{A}^{1 / 2}$. Оставляя в стороне вопрос о том, лежит ли $f=x_{1}+\alpha / \theta$ (а стало быть, и координатная функция $x_{1}$ ) в области определения оператора $\bar{A}$, можно заключить, что равенство $D f=\bar{A} f$ невозможно.

Эти рассуждения объясняют следующий кажущийся парадокс. Если рассматривать правую часть формулы (16) как генератор диффузионного процесса (аналогично конечномерной ситуации), то получится, что вектор сноса в точке $x=(0,0, \ldots)$ равен $(-\alpha,-\alpha, \ldots)$, т. е. направлен «во вне» симплекса $\bar{\nabla}_{\infty}$. Но действие генератора $\bar{A}$ на координатные функции $x_{1}, x_{2}, \ldots$ заведомо не дается правой частью формулы (16). Поэтому в нашей ситуации нельзя интерпретировать коэффициенты при первых производных в (16) как координаты вектора сноса.

5.2. Предгенератор $\boldsymbol{A}$ в моментных координатах. Сначала приведем следующее определение.

Пусть дана коммутативная алгебра. Будем говорить, что каждый оператор умножения на элемент этой алгебры имеет нулевой порядок. Будем говорить, что оператор $Q$ имеет порядок $n \geqslant 1$, если его коммутатор с любым оператором умножения на элемент алгебры имеет порядок $n-1$. 
Лемма 5.5. Предгенератор А является оператором второго порядка в алгебре $\mathscr{F}$.

Доказательство. Требуется показать, что $\left[\left[\left[A, H_{1}\right], H_{2}\right], H_{3}\right] f(x)=0$ для всех $h_{1}, h_{2}, h_{3}, f \in \mathscr{F}$ и $x \in \bar{\nabla}_{\infty}$, где $H_{j}$ означает оператор умножения на $h_{j}$, $j=1,2,3$.

Из п. (2) замечания 5.3 следует, что это равенство выполнено для всех $x \in$ $\nabla_{\infty}$. Действительно, в этом случае оператор $A$ действует согласно правой части формулы (16), и в силу линейности достаточно сослаться на то, что

$$
\left[\left[\left[\partial^{2} / \partial x_{i} \partial x_{j}, H_{1}\right], H_{2}\right], H_{3}\right] f(x)=0 \quad \text { и } \quad\left[\left[\left[\partial / \partial x_{i}, H_{1}\right], H_{2}\right], H_{3}\right] f(x)=0
$$

для всех $1 \leqslant i, j<\infty$.

Для произвольных $x \in \bar{\nabla}_{\infty}$ утверждение верно по непрерывности.

В силу того что моментные координаты $q_{1}, q_{2}, \ldots$ являются алгебраически независимыми образующими алгебры $\mathscr{F}$, а оператор $A$ имеет второй порядок, его действие в $\mathscr{F}$ полностью определяется значениями $A 1, A q_{i}, A\left(q_{i} q_{j}\right), i, j=$ $1,2, \ldots$ Ясно, что $q_{i} q_{j}=q_{i+j+1}+\mathbf{m}_{(i+1, j+1)}^{\circ}, i \geqslant j$. Используя (14) (или, что эквивалентно, (16) вместе с замечаниями 5.2 и 5.3), получаем, что

$$
\begin{aligned}
A 1 & =0, \\
A q_{i} & =-(i+1)(i+\theta) q_{i}+(i+1)(i-\alpha) q_{i-1}, \\
A\left(q_{i} q_{j}\right) & =q_{i} A q_{j}+q_{j} A q_{i}+2(i+1)(j+1)\left(q_{i+j}-q_{i} q_{j}\right),
\end{aligned}
$$

где принято соглашение $q_{0}=1$.

Заметим, что правая часть формулы (2) действует на функции $1, q_{i}$ и $q_{i} q_{j}$, $i, j=1,2, \ldots$, точно так же, как выписано выше, и поэтому оператор $A$ можно записать в виде (2). Данный вид предгенератора $A$ позволяет показать, что почти все траектории процесса $\mathbf{X}_{\alpha, \theta}(t)$ непрерывны. Это можно доказать так же, как следствие 6.4 и теорему 7.1 в [2].

5.3. Вырожденные значения параметров. Отметим, что происходит, когда параметры $\alpha$ и $\theta$ лежат в вырожденной серии. Рассмотрим конечномерный упорядоченный симплекс

$$
\nabla_{N}:=\left\{\left(x_{1}, \ldots, x_{N}\right) \in \mathbb{R}^{\mathbb{N}}: x_{1} \geqslant \cdots \geqslant x_{N} \geqslant 0, \sum_{i=1}^{N} x_{i}=1\right\}, \quad N=2,3, \ldots
$$

Можно мыслить $\nabla_{N}$ как подмножество в $\nabla_{\infty} \subset \bar{\nabla}_{\infty}$. Мера $\operatorname{PD}(\alpha, \theta)$ сосредоточена на $\nabla_{\infty}$ при всех значениях $\alpha$ и $\theta$, лежащих в основной или вырожденной серии.

Предложение 5.6 [25, с. 62]. При $\alpha=-\beta<0$ u $\theta=N \beta$ мера $\mathrm{PD}(-\beta, N \beta)$ сосредоточена на $\nabla_{N} \subset \bar{\nabla}_{\infty}$. Она совпадает с мерой на $\nabla_{N}$, которая имеет следуюшую плотность относительно меры Лебега:

$$
\operatorname{PD}(-\beta, N \beta)(d x)=\frac{N ! \Gamma(N \beta)}{(\Gamma(\beta))^{N}} x_{1}^{\beta-1} \cdots x_{N}^{\beta-1} d x_{1} \cdots d x_{N-1} .
$$

Кроме того, при $\beta \rightarrow+0, N \rightarrow+\infty$ и $N \beta \rightarrow \eta>0$ имеет место слабая сходимость мер на $\bar{\nabla}_{\infty}: \operatorname{PD}(-\beta, N \beta) \rightarrow \operatorname{PD}(0, \eta)$. 
Оказывается, данный результат распространяется и на процесс $\mathbf{X}_{\alpha, \theta}(t)$ на $\bar{\nabla}_{\infty}$. Для начала напомним, какие диффузионные процессы рассматриваются на конечномерном симплексе $\nabla_{N}$.

Нас будут интересовать процессы, называемые в [4] диффузионными аппроксимациями популяиионной модели Райта-Фишера с симметричной мутаиией, которые рассматривались также, например, в [30] и [12]. Детальное построение более общих конечномерных диффузий, связанных с популяционной генетикой, см. также в [5, гл. 10].

Пусть $\mathscr{F}_{N}=\mathbb{R}\left[q_{1}, \ldots, q_{N-1}\right]$ - коммутативная алгебра с единицей, свободно порожденная моментными координатами $q_{k}(x)=\sum_{i=1}^{N} x_{i}^{k+1}$ на $\nabla_{N}, k=$ $1, \ldots, N-1$. Ясно, что $\mathscr{F}_{N} \subset C\left(\nabla_{N}\right)$ - плотная подалгебра. Для каждого значения параметра $\eta>0$ определим в ней оператор

$$
A_{N, \eta}:=\sum_{i=1}^{N} x_{i} \frac{\partial^{2}}{\partial x_{i}^{2}}-\sum_{i, j=1}^{N} x_{i} x_{j} \frac{\partial^{2}}{\partial x_{i} \partial x_{j}}+\frac{\eta}{N-1} \sum_{i=1}^{N}\left(1-N x_{i}\right) \frac{\partial}{\partial x_{i}} .
$$

Заметим, что этот оператор (в отличие от своего бесконечномерного аналога из разд. 5.1) можно сразу определить по той же формуле на более широком подпространстве в $C\left(\nabla_{N}\right)$. Оно состоит из дважды непрерывно дифференцируемых функций с некоторыми граничными условиями, см. [4, (2.8)]. Обозначим это расширение через $\widetilde{A}_{N, \eta}$.

Предложение 5.7 [4]. (1) Onератор $\widetilde{A}_{N, \eta}$ (а стало бъть, и $\left.A_{N, \eta}\right)$ замъкаем в $C\left(\nabla_{N}\right)$. Обозначим через $\bar{A}_{N, \eta}$ его замыкание (а стало быть, и замыкание onepamopa $\left.A_{N, \eta}\right)$.

(2) Замыкание $\bar{A}_{N, \eta}$ порождает дифбузионный процесс (m.е. строго марковское семейство с непрерьвными траекториями) на $\nabla_{N}$.

(3) Этот процесс сохраняет меру $\mathrm{PD}\left(-\frac{\eta}{N-1}, \frac{N \eta}{N-1}\right)$, определенную выше, $u$ обратим относительно нее.

Обозначим этот процесс через $\mathbf{Y}_{N, \eta}(t)$.

Предложение 5.8 [4, теорема 2.5]. При $N \rightarrow \infty$ процессы $\mathbf{Y}_{N, \eta}(t)$ сходятся $\Re^{1)} \kappa$ прочессу $\mathbf{X}_{0, \eta}(t)$, построенному в $\S 4$.

Оказывается, что можно рассматривать процесс $\mathbf{X}_{\alpha, \theta}(t)$, построению и изучению которого посвящена данная работа, и при вырожденных значениях параметров. Для начала опишем, как надо модифицировать марковские цепи вверх /вниз.

Пусть $\mathbb{K}(N)$ состоит из всех диаграмм $\lambda \in \mathbb{K}$, для которых $\ell(\lambda) \leqslant N$. Это снова градуированный граф. Пусть $\alpha=-\beta, \theta=N \beta$. В этом случае каждая из мер $M_{n}$, определенных в разд. 1.3 , положительна всюду на $\mathbb{K}_{n}(N):=\mathbb{K}(N) \cap \mathbb{K}_{n}$. Таким образом, можно рассматривать цепи вверх вниз на $\mathbb{K}_{n}(N)$ аналогично разд. 1.2. Роль алгебры $\Lambda$ будет играть $\Lambda_{N}$ - алгебра симметрических функций от $N$ переменных. Ясно, что $\Lambda_{N} / I \cong \mathscr{F}_{N}$, где $I=\left(p_{1}-1\right) \Lambda_{N}$ - идеал алгебры $\Lambda_{N}$ (см. §2). Вложения $\iota_{n}$, определенные в разд. 2.3 , теперь отображают $\mathbb{K}_{n}(N)$ B $\nabla_{N}$.

1) В определенном смысле, который объясняется в [4, теорема 2.5]. 
Предложение 5.9. (1) Пусть $T_{n}(N)$ - производящий оператор $n$-й марковской иепи вверх/вниз. В том же смысле, что и в лемме 4.1, имеет место сходимость

$$
\lim _{n \rightarrow \infty} n^{2}\left(T_{n}(N)-\mathbf{1}\right) f=A_{N, \beta(N-1)} f \quad \text { для всех } f \in \mathscr{F}_{N} .
$$

(2) Дискретные полугруппъ $\left\{\mathbf{1}, T_{n}(N), T_{n}^{2}(N), \ldots\right\}$ при $n \rightarrow \infty$ сходятся (в том же смысле, что в предложении 4.3) $\kappa$ непрерывной полугруппе в пространстве $C\left(\nabla_{N}\right)$, порожденной оператором $\bar{A}_{N, \beta(N-1)}$.

Таким образом, естественно считать, что процесс $\mathbf{X}_{-\beta, N \beta}(t)$ совпадает с $\mathbf{Y}_{N, \beta(N-1)}(t)$, т. е. является конечномерным диффузионным процессом в $\nabla_{N}$ с предгенератором $A_{N, \beta(N-1)}: \mathscr{F}_{N} \rightarrow \mathscr{F}_{N}$. Значит, диффузии на конечномерных симплексах, рассматриваемые в [4] и во многих других работах, являются частным случаем двухпараметрического семейства диффузий $\mathbf{X}_{\alpha, \theta}(t)$ на $\bar{\nabla}_{\infty}$.

Предложение 5.8 можно интерпретировать как сходимость (в смысле [4, теорема 2.5]) процессов $\mathbf{X}_{-\beta, N \beta}(t)$ на $\nabla_{N}$ к процессу $\mathbf{X}_{0, \eta}(t)$ на $\bar{\nabla}_{\infty}$ при $\beta \rightarrow+0$, $N \rightarrow+\infty$ и $N \beta \rightarrow \eta>0$. Эта сходимость усиливает второе утверждение предложения 5.6 о сходимости инвариантных мер.

\section{ЛитерАТУрА}

[1] J. Bertoin, A second order SDE for the Langevin process reflected at a completely inelastic boundary, J. Eur. Math. Soc. (JEMS), 10:3 (2008), 625-639.

[2] A. Borodin, G. Olshanski, Infinite-dimensional diffusions as limits of random walks on partitions, Probab. Theory Related Fields, 144:1-2 (2009), 281-318; http://arxiv.org/abs/0706.1034v1.

[3] R. Dong, A. Gnedin, J. Pitman, Exchangeable partitions derived from Markovian coalescents, Ann. Appl. Probab., 17:4 (2007), 1172-1201; http://arxiv.org/abs/ math/0603745.

[4] S. N. Ethier, T. G. Kurtz, The infinitely-many-neutral-alleles diffusion model, Adv. Appl. Probab., 13:3 (1981), 429-452.

[5] S. N. Ethier, T. G. Kurtz, Markov Processes. Characterization and Convergence, Wiley-Interscience, New York, 1986.

[6] S. N. Ethier, Eigenstructure of the infinitely-many-neutral-alleles diffusion model, J. Appl. Probab., 29:3 (1992), 487-498.

[7] W. J. Ewens, Mathematical Population Genetics, Springer-Verlag, Berlin-New York, 1979.

[8] J. Fulman, Stein's method and Plancherel measure of the symmetric group, Trans. Amer. Math. Soc., 357:2 (2005), 555-570.

[9] J. Fulman, Commutation relations and Markov chains, Probab. Theory Related Fields, 144:1-2 (2009), 99-136; http://arxiv.org/abs/0712.1375.

[10] S. Feng, F.-Y. Wang, A class of infinite dimensional diffusion processes with connection to population genetics, Journal Appl. Probab., 44:4 (2007), 938-949; http://arxiv.org/abs/0711.1887v1..

[11] A. Gnedin, J. Pitman, Regenerative partition structures, Electronic J. Combin., 11:2 (2005), research paper 12.

[12] R. C. Griffiths, A transition density expansion for a multi-allele diffusion model, Adv. Appl. Probab., 11:2 (1979), 310-325. 
[13] Ц. Игнатов, Об одной константе, возникающей в асимптотической теории симметрических групп, и о мерах Пуассона-Дирихле, Теория вероятн. и ее прим., 27:1 (1982), 129-140..

[14] S. Karlin, J. McGregor, The number of mutant forms maintained in a population, in: Proc. 5th Berkeley Symposium on Math Statist. Prob., vol. 4, 1967, 415-438.

[15] S. V. Kerov, Asymptotic representation theory of the symmetric group and its applications in analysis, Amer. Math. Soc., Providence, RI, 2003; http://www.mathsoc. spb.ru/preprint/2001/01-04.ps.gz.

[16] S. Kerov, A. Okounkov, G. Olshanski, The boundary of Young graph with Jack edge multiplicities, Intern. Math. Res. Notices, 1998:4 (1998), 173-199.

[17] J. F. C. Kingman, Random discrete distributions, J. R. Statist. Soc. B, 37 (1975), $1-22$.

[18] J. F. C. Kingman, The population structure associated with the Ewens sampling formula, Theoret. Population Biology, 11:2 (1977), 274-283.

[19] J. F. C. Kingman, Random partitions in population genetics, Proc. Roy. Soc. London, Ser. A, 361:1704 (1978), 1-20.

[20] И. Макдональд, Симметрические функиии и многочлень Холла, Мир, М., 1985.

[21] M. Perman, J. Pitman, M. Yor, Size-biased sampling of Poisson point processes and excursions, Probab. Theory Related Fields, 92:1 (1992), 21-39.

[22] J. Pitman, The two-parameter generalization of Ewens' random partition structure, Technical report 345, Dept. Statistics, U. C. Berkeley, 1992, http://www.stat. berkeley.edu/tech-reports/

[23] J. Pitman, Exchangeable and partially exchangeable random partitions, Probab. Theory Related Fields, 102:2 (1995), 145-158.

[24] J. Pitman, Random discrete distributions invariant under size-biased permutation, Adv. Appl. Probab., 28:2 (1996), 525-539.

[25] J. Pitman, Combinatorial Stochastic Processes (Ecole d'Eté de Probabilités de SaintFlour XXXII-2002), Lecture Notes in Math., vol. 1875, Springer-Verlag, Berlin, 2006; http://works. bepress.com/jim_pitman/1.

[26] J. Pitman, M. Yor, The two-parameter Poisson-Dirichlet distribution derived from a stable subordinator, Ann. Probab., 25 (1997), 855-900.

[27] B. Schmuland, A result on the infinitely many neutral alleles diffusion model, J. Appl. Probab., 28:2 (1991), 253-267.

[28] А. М. Вершик, А. А. Шмидт, Пределънъе мерь, возникающие в асимптотической теории симметрических групn, I u II, Теория вероятн. и ее прим., 22:1 (1977), 72-88; 23:1 (1978), 42-54.

[29] G. A. Watterson, Reversibility and the age of an allele. I. Moran's infinitely many neutral alleles model, Theoret. Population Biology, 10:3 (1976), 239-253.

[30] G. A. Watterson, The stationary distribution of the infinitely-many-neutral-alleles diffusion model, J. Appl. Probab., 13:4 (1976), 639-651.

Институт проблем передачи информации

им. А. А. Харкевича РАН

Поступило в редакцию

e-mail: lenia.petrov@gmail.com 8 октября 2008 г. 